
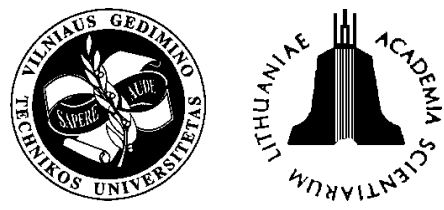

\title{
THE DETERMINATION OF THE ENDOGENOUS MARKET PENETRATION AND COMPLIANCE RATE OF ADVANCED TRAVELER INFORMATION SYSTEMS
}

\author{
Shu Guang Li \\ Dept of Automation, School of Electrical Engineering, Xi'an Jiao Tong University, \\ Xi'an 710049, People's Republic of China. E-mail: lxlsg@sohu.com \\ Received 2004-01-29; accepted 2004-04-15
}

\begin{abstract}
We consider a specific ATIS which objective is to reduce drivers' travel cost uncertainty with recurrent network congestion through the provision of traffic information. Most of the studies premise of the use of a static model for an essentially dynamic problem. We propose a multi-user stochastic dynamic route and departure time model for modeling interactions among travelers. We propose a nested-logit model for the determination of the market penetration and compliance rate of ATIS. The upper level is for market penetration; the lower level is for compliance rate. Then an iterative algorithm is proposed for the determination of the endogenous market penetration and compliance rate of ATIS and the equilibrium network flow pattern. Finally, the model and algorithm are tested in a simple network.
\end{abstract}

Keywords: advanced traveler information systems; market penetration; compliance rate; nested logit model.

\section{Introduction}

As the main part of intelligent transportation systems (ITS), Advanced traveler information systems that have experienced rapid development in the past decades are generally believed to be an efficient means for improving individual traveler's trip planning, alleviating traffic congestion and enhancing traffic network performance. There are many researches into modelling and evaluating the effect of ATIS on travelers' and transportation systems in order to determine the feasibility, risks and benefits of such technology. Up to now researchers use many ways such as route choice survey (Abdel. Aty, M. A etc, 1997, E. Hato, etc, 1999), field deployments (Tsuji etc, 1985), laboratory experiments (Yang 1993, Reddy 1995), computer simulation (Emmerink 1995, Mahmassani 1994, 1999) and analytical model (Yang Hai 1998, 999, Ya Feng Yin 2003, H. LO 2002a, William 2003).

The route choice of travelers with ATIS and without ATIS is the most important aspect of ATIS. The difference of the information quality achieved by travelers will lead to various route choice behaviors. Previous studies have considered the travelers with ATIS follow SO, deterministic user equilibrium or Stochastic user equilibrium (smaller perception uncertainty), the travelers without ATIS follow SUE (higher perception uncertainty) (Kanafani and Al-Deek, 1991; Van
Vuren\&Walting 1991; Benneet, 1993; Yang, 1998; Lo et al., 1999; Yang and Meng, 2001; Yin and Yang, 2003).

The market penetration of ATIS defined as the proportion of vehicles (travelers) equipped with ATIS has widely recognized as an important factor to determine the actual advantage of ATIS implementation. The exogenous market penetration was used for the benefits of ATIS in some previous studies (Yang 1999, Emmerink 1995), regardless of the individual's trip cost or potential travel cost saving. Yang (1998) was first to propose the endogenous market penetration model. Yang (2001) further modeled the time line of the growth of market penetration to reach final stationary equilibrium based on a modified logistic type growth model. Similar studies were carried out by Lo and Szeto (1999, 2002a), Yin (2003).

Compliance is one important aspect of traveler's behaviors that has received little attention. Previous researches have assumed perfect compliance of the travelers with ATIS. However, although ATIS are intended to provide accurate real-time traffic information to travelers, it is doubtful whether travelers would all comply with these systems. There are many factors that affect traveler's compliance with ATIS such as driver individual characteristics, reliability of ATIS and so on. Yin (2003) proposed a multiple behavior equilibrium model to determine the equilibrium market penetration and compliance rate. 
However, most of the previous models for assessing the effect of ATIS are static. These models generally don't permit the study of other ATIS impacts such as changes in departure time, dynamic queuing scenario and so on, which may bias the analysis. Emmerink 1995has contributed substantially in studying the economic impacts of driver information system by using microscopic simulations. Mahmassani etc (1994) used DYNASMART model to assess the effect of ATIS. Lo (2002) developed a dynamic traffic assignment model, compared and contrasted the static versus the dynamic modeling paradigms for the benefits of ATIS. William and Huang (2003) proposed a multi-class dynamic user equilibrium model with ATIS, that considers the departure time and network queue conditions and so on. The similar studies were carried out by B. Ran (1996), Hong K. Lo (1996). However they didn't consider the endogenous market penetration and compliance rate.

In this paper we extend Ying's (2003) static ATIS model to a dynamic one by taking advantage of the dynamic route choice platform developed in (H. Khen, 1998). We assume that equipped and complied drivers will follow a stochastic dynamic simultaneous route and departure time equilibrium behavior with less travel cost uncertainty, unequipped and non-complied drivers follow stochastic dynamic simultaneous route and departure time equilibrium behavior with more travel cost uncertainty. Thus, two specific classes of travelers are considered in the dynamic network equilibrium model, as illustrated in Fig 1. In view of the fact that travel cost is the most important factor to influence the traveler's route and departure time choice, it is rational to assume that actual travel cost saving will ascertain whether or not for a traveler to purchase an ATIS device and, if equipped, whether or not to follow ATIS advice. The nested-logit model is used in solving the equilibrium market penetration and compliance rate of ATIS, the upper level depicts traveler's purchase behavior, and the lower level depicts compliance behavior of equipped travelers. With these considerations we propose an iterative procedure to calculate the endogenous equilibrated market penetration and compliance rate of ATIS.

The other parts of this paper are organized as follows. In section 2, we propose a multi-user dynamic simultaneous route/departure time equilibrium model to described route and departure time choices of the equipped and complied and unequipped and non-complied travelers. In section 3 the endogenous market penetration and compliance rate of ATIS are determined simultaneously by using the nested-logit model. In section 4, an iterative algorithm is developed and illustrated with a numerical example in section.

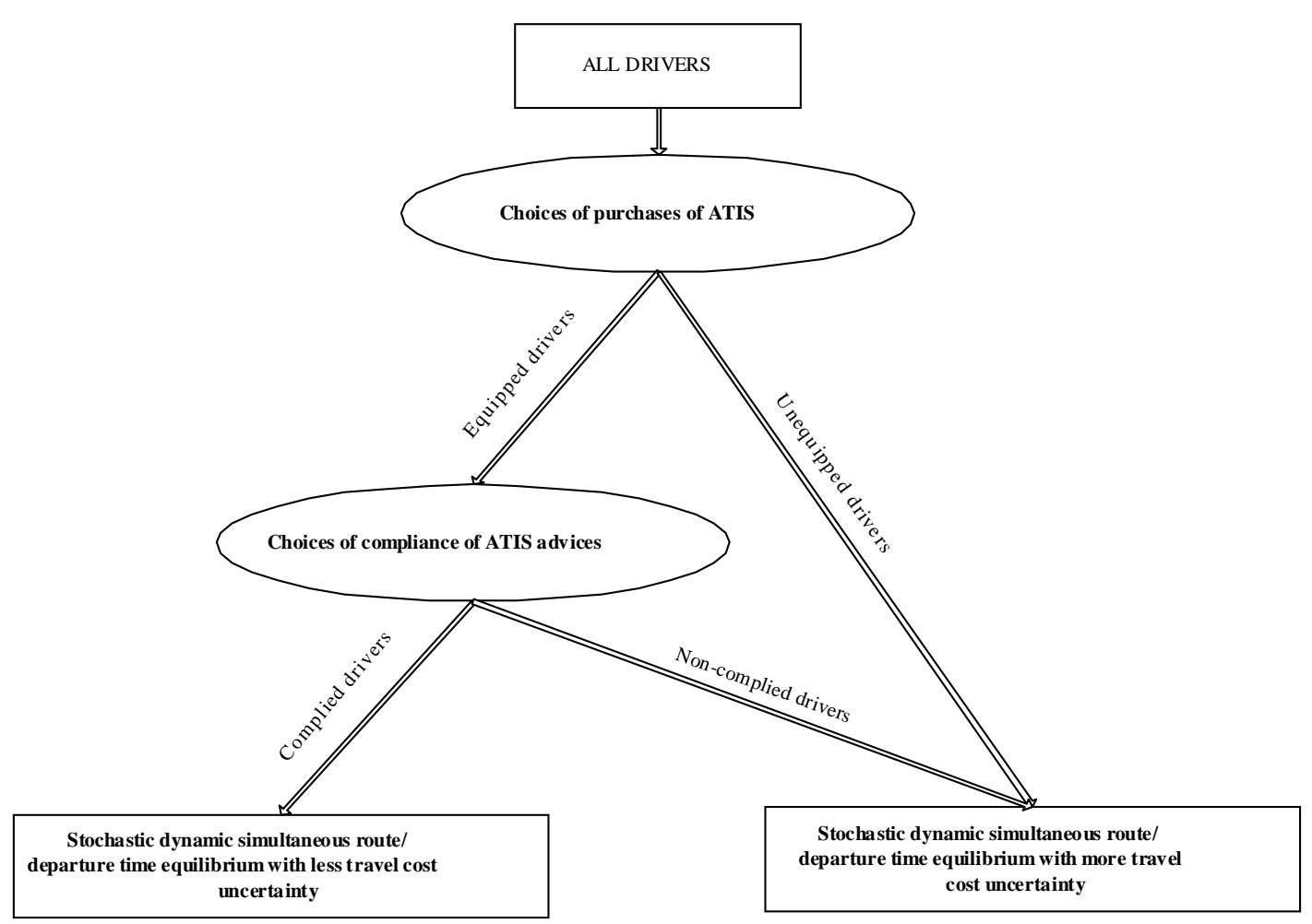

Fig 1. Multiple user behaviors in ATIS environment 


\section{Dynamic multi-user VI model}

\subsection{Multi-user stochastic dynamic equilibrium} models

Consider a network $\mathrm{G}=(\mathrm{N} ; \mathrm{A})$, where $\mathrm{N}$ is the set of nodes and $\mathrm{A}$ is the set of links in the network. Let $a$ denote a link of the network connecting a pair of nodes $(i, j)$ and let $p$ denote a path that consists of a series of directed link $\left(a_{1}, a_{2}, \ldots, a_{\mathrm{n}}\right)$ between origin $r$ and destination $s$. Let RS denote the set of all OD pairs in the network. $P_{\mathrm{rs}}$ denotes the set of routes between OD pair $r s \bar{I}$ RS and the entire set of paths in the network by $P$. The studies horizon is discretized into $m$ intervals of length $\delta$ such that $T=m \cdot \delta$. Here, we assume the study horizon is enough long to ensure all traveler can exit from the network after the time $T$. On the other hand, it is also assumed that the value of $\delta$ is small enough so that the discrete-time model can approximate its continuous time counterpart.

\subsection{Stochastic dynamic user equilibrium}

In most of previous multi-class dynamic network model (Bin. R 1996, Hong. K 1996Williams and Huang 2003 etc), the equipped travelers who may receive the real-time perfect traffic information are assumed to make travel choice in a deterministic dynamic user equilibrium manner. In reality traffic information is rarely perfect. There are many difficulties for estimating the current traffic information and predicting the future traffic information in current traffic technology condition. Moreover, a greater number of the equipped travelers might select the best alternatives (from their individual point of view) and consequently the equipped travelers with similar preferences will tend to concentrate on the same routes during the same departure time. Thus, more perfect information could potentially generate higher levels of traffic congestion. The traffic manager and individual travelers don't hope this condition happen. For these reasons we model the travel choice behavior of the equipped travelers as following the principle of stochastic dynamic user equilibrium. The traffic information quality is reflected by the parameter of general travel cost perception variation. Better information qualities have lower travel cost variation. The unequipped travelers in making travel choices, in particular, route and departure time choice, according to the past experiences that don't capture better information than the equipped travelers, follow other stochastic dynamic user equilibrium with a higher travel cost variation.

$$
\hat{f}_{p}^{r s}(k), \tilde{f}_{p}^{r s}(k) \text { the inflow rate of the equipped }
$$

and complied and unequipped and non-complied travelers entering the path $p$ between origin $r$ and destination $s$ during time interval $k$, $\hat{f}, \tilde{f}$ the set of $\left\{\hat{f}_{m}^{r s}(k), \forall r s, p, k\right\}$

$\left\{\tilde{f}_{p}^{r s}(k), \forall r s, p, k\right\}$,

$\hat{q}^{r s}, \tilde{q}^{r s}$ the demand of equipped and complied and unequipped and non-complied travelers between origin $r$ and destination $s$,

$\hat{P}_{p}^{r s}(k), \widetilde{P}_{p}^{r s}(k)$ the proportion of equipped and complied and unequipped and non-complied travelers between origin $r$ and destination $s$ selecting path $p$ and departure time $k$.

$\hat{\theta}$ the parameter representing travel cost variation of equipped and complied travelers.

$\tilde{\theta}$ the parameter representing travel cost variation of unequipped and non-complied travelers.

The equipped and complied travelers follow the stochastic dynamic simultaneous route and departure time equilibrium (SUE-SRD), expressed as

$$
\hat{f}_{p}^{r s}(k)=\hat{P}_{p}^{r s}(k) \hat{q}^{r s} \quad \forall r s, p, k
$$

where

$$
\hat{P}_{p}^{r s}(k)=\frac{\exp \left(-\hat{\theta} c_{p}^{r s}(k)\right)}{\sum_{p} \sum_{k} \exp \left(-\hat{\theta} c_{p}^{r s}(k)\right)} \quad \forall r s, p, k
$$

where $\hat{\theta}$ is the parameter representing general travel cost perception variation of the equipped and complied travelers. A higher $\hat{\theta}$ means smaller travel cost variation and better information quality.

The logit-based SUE-SRD of the equipped and complied travelers can be expressed as:

$$
\begin{aligned}
& \hat{C}_{p}^{r s}\left(k, f^{*}\right)\left\{\begin{array}{cc}
\hat{c}_{\text {min }}^{r s} \text { if } & \hat{f}_{p}^{r s^{*}}(k)>0 \\
>\hat{c}_{\text {min }}^{r s} & \text { otherwise }
\end{array} \quad \forall r s, k, p\right. \\
& \sum_{p} \sum_{k} \hat{f}_{p}^{r s^{*}}(k)=\frac{\hat{q}^{r s}}{\delta} \quad \forall r s \\
& \hat{C}_{p}^{r s}\left(k, f^{*}\right)=c_{p}^{r s}(k)+\frac{1}{\hat{\theta}} \ln \hat{f}_{p}^{r s^{*}}(k) \quad \forall r s, k, p \\
& \hat{f}_{p}^{r s^{*}}(k) \geq 0 \quad \forall r s, k, p,
\end{aligned}
$$

where $\hat{c}_{\min }^{r s}$ is the minimum perceived unit travel cost of the equipped and complied travelers between origin $r$ and destination $s, \quad \hat{c}_{\text {min }}^{r s}=\min \left\{\hat{C}_{p}^{r s}(k, f)^{*}, \forall p, k\right\}$. $\hat{C}_{p}^{r s}\left(k, f^{*}\right)$ is perceived unit travel cost incurred by the equipped and complied travelers entering path $p$ between origin $r$ and destination $s$ during time interval $k$. Equation (38) represents the flow conservation of the equipped and complied travelers between origin $r$ and 
destination $s$ and equation (40) represents the nonnegativity of all path inflow rate.

For the equipped and complied travelers and for each origin-destination (OD) pair, the perceived path travel costs experienced for the equipped and complied travelers, regarding the departure time, is equal and minimum, and less than (or equal to) the perceived path travel costs for the equipped and complied travelers on any unused route.

The above SUE-SRD equilibrium condition of the equipped and complied travelers can be expressed by finite dimensional variational inequality formulation.

Find a vector $\hat{f}^{*} \in \Omega$ if and only if it satisfies

$$
\sum_{r s} \sum_{p} \sum_{k} \hat{C}_{p}^{r s}\left(k, f^{*}\right)\left(\hat{f}_{p}^{r s}(k)-\hat{f}_{p}^{r s^{*}}(k)\right) \geq 0 \forall \hat{f} \in \hat{\Omega},(7)
$$

where $\hat{\Omega}$ is a closed convex.

$$
\hat{\Omega}=\left\{\hat{f} \mid \sum_{p} \sum_{k} \hat{f}_{p}^{r s}(k)=\frac{\hat{q}^{r s}}{\delta}, \quad \hat{f}_{p}^{r s}(k) \geq 0, \forall r s\right\}
$$

formation (7) can be expressed as a standard form

$$
\left\langle\hat{C}\left(\hat{f}^{*}\right), \hat{f}-\hat{f}^{*}\right\rangle \geq 0 \quad \forall \hat{f} \in \hat{\Omega},
$$

where $\langle\cdot$,$\rangle denoted the inner product of Euclidean$ space.

The treatment of the unequipped and non-complied travelers is identical. Without loss of generality, one may write:

$$
\tilde{f}_{p}^{r s}(k)=\tilde{P}_{p}^{r s}(k) \cdot \tilde{q}^{r s} \quad \forall r s, p, k,
$$

where

$$
\tilde{P}_{p}^{r s}(k)=\frac{\exp \left(-\tilde{\theta} \cdot c_{p}^{r s}(k)\right)}{\sum_{p} \sum_{k} \exp \left(-\tilde{\theta} \cdot c_{p}^{r s}(k)\right)} \forall r s, p, k,
$$

where $\tilde{\theta}$ expresses the travel cost perception variation of the unequipped and non-complied travelers that can be interpreted as their familiarity of the network condition or the past experiences.

The logit-based SUE-SRD of the unequipped and non-complied travelers can be expressed as:

$$
\begin{aligned}
& \tilde{C}_{p}^{r s^{*}}\left(k, f^{*}\right)\left\{\begin{array}{l}
=\tilde{c}_{\text {min }}^{r s} \text { if } \tilde{f}_{p}^{r s^{*}}(k)>0 \\
>\tilde{c}_{\text {min }}^{r s} \text { otherwise }
\end{array}, \forall r s, k, p ;\right. \\
& \sum_{p} \sum_{k} \tilde{f}_{p}^{r s^{*}}(k)=\frac{\tilde{q}^{r s}}{\delta} \quad \forall r s ;
\end{aligned}
$$

$$
\begin{gathered}
\tilde{C}_{p}^{r s^{*}}\left(k, f^{*}\right)=c_{p}^{r s}(k)+\frac{1}{\tilde{\theta}} \ln \tilde{f}_{p}^{r s^{*}}(k) \quad \forall r s, k, p ; \\
\tilde{f}_{p}^{r s^{*}}(k) \geq 0 \quad \forall r s, k, p .
\end{gathered}
$$

The above SUE-SRD equilibrium condition of the unequipped and non-complied travelers can be expressed by finite dimensional variational inequality formulation.

Find a vector $\hat{f}^{*} \in \Omega$ if and only if it satisfies

$$
\begin{aligned}
& \sum_{r s} \sum_{p} \sum_{k} \tilde{C}_{p}^{r s^{*}}\left(k, f^{*}\right)\left(\tilde{f}_{p}^{r s}(k)-\tilde{f}_{p}^{r s^{*}}(k)\right) \geq 0 \\
& \forall \tilde{f} \in \tilde{\Omega},
\end{aligned}
$$

where $\hat{\Omega}$ is a closed convex.

$$
\tilde{\Omega}=\left\{\tilde{f} \mid \sum_{p} \sum_{k} \tilde{f}_{p}^{r s}(k)=\frac{\tilde{q}^{r s}}{\delta}, \quad \tilde{f}_{p}^{r s}(k) \geq 0, \forall r s\right\} .
$$

The VI formation (15) can be expressed as a standard form

$$
\left\langle\tilde{C}\left(\tilde{f}^{*}\right), \tilde{f}-\tilde{f}^{*}\right\rangle \geq 0 \quad \forall \tilde{f} \in \tilde{\Omega} .
$$

\subsection{The composite VI formulation}

The composite VI problem that integrates the VI (7) with VI (15) is equivalent to the above user equilibrium condition (3) and (11).

The composite VI model can be formulated as follows:

Find a vector $\left(\hat{f}^{*} \in \hat{\Omega}, \tilde{f}^{*} \in \tilde{\Omega}\right)$ that is a multi-user stochastic dynamic user equilibrium pattern if and only if it satisfies the VI problem:

$$
\begin{aligned}
& \sum_{r s} \sum_{p} \sum_{k} \tilde{C}_{p}^{r s}\left(k, f^{*}\right)\left(\tilde{f}_{p}^{r s}(k)-\tilde{f}_{p}^{r s^{*}}(k)\right)+ \\
& \sum_{r s} \sum_{p} \sum_{k} \hat{C}_{p}^{r s^{*}}\left(k, f^{*}\right)\left(\hat{f}_{p}^{r s}(k)-\hat{f}_{p}^{r s^{*}}(k)\right) \geq 0 \\
& \forall \hat{f} \in \hat{\Omega}, \forall \tilde{f} \in \tilde{\Omega},
\end{aligned}
$$

where $\hat{\Omega}$ and $\tilde{\Omega}$ are the sets of all feasible path inflow rate with all departure time associated with the equipped and complied and unequipped and non-complied travelers.

The composite VI (17) can be expressed as a standard form:

$$
\begin{aligned}
& \left\langle\tilde{C}\left(\tilde{f}^{*}\right), \tilde{f}-\tilde{f}^{*}\right\rangle+\left\langle\hat{C}\left(\hat{f}^{*}\right), \hat{f}-\hat{f}^{*}\right\rangle \geq 0 \\
& \forall \hat{f} \in \hat{\Omega} \quad \forall \tilde{f} \in \tilde{\Omega} .
\end{aligned}
$$




\subsection{Dynamic network model}

In this section we use the multi-user dynamic network model proposed by H.K. Chen (1998).

Flow propagation constraint is:

$$
\begin{aligned}
& \hat{u}_{a p k}^{r s}(t)=\hat{f}_{p}^{r s}(k) \delta_{a p k}^{r s}(t) \quad \forall r s, a \in p, k ; \\
& \tilde{u}_{a p k}^{r s}(t)=\tilde{f}_{p}^{r s}(k) \delta_{a p k}^{r s}(t) \quad \forall r s, a \in p, k ; \\
& \sum_{t} \delta_{a p k}^{r s}(t)=1 \quad \forall r s, a \in p, k ; \\
& \delta_{a p k}^{r s}(t)=\{0,1\} \quad \forall r s, a \in p, k,
\end{aligned}
$$

where $\hat{u}_{a p k}^{r s}(t)$ and $\tilde{u}_{a p k}^{r s}(t)$ express the path flow rate of the equipped and complied and unequipped and noncomplied travelers entering link $a$ during time interval $k$ between OD pair $r s$, respectively. $\delta_{a p k}^{r s}(t)$ represents the flow propagation factor.

Definitional constraint is:

$$
\begin{aligned}
& u_{a}(k)=\sum_{r s} \sum_{p} \sum_{k}\left(\hat{f}_{p}^{r s}(k)+\tilde{f}_{p}^{r s}(k)\right) \delta_{a p k}^{r s}(t) ; \\
& t_{p}^{r s}(k)=\sum_{a} \sum_{t} t_{a}(k) \delta_{a p k}^{r s}(t) \forall r s, p, k ; \\
& c_{p}^{r s}(k)=\alpha t_{p}^{r s}(k)+s c h_{p}^{r s}(k) \forall r s, p, k,
\end{aligned}
$$

where $u_{a}(k)$ expresses the link inflow rate entering link $a$ during time interval $k . t_{p}^{r s}(k)$ expresses actual path travel time experienced by the travelers entering the path $p$ between OD pair $r s$ during time interval $k$. $c_{p}^{r s}(k)$ expresses actual path travel cost experienced by the travelers entering the path $p$ between OD pair $r s$ during time interval $k . \alpha$ is the unit cost of travel time, $\operatorname{sch}_{p}^{r s}(k)$ expresses the schedule delay costs of arriving times at destination.

$$
\operatorname{sch}_{p}^{r s}(k)=\left\{\begin{array}{cc}
\beta\left[k_{r s}^{*}-\Delta_{r s}-k-t_{p}^{r s}(k)\right] \text { if } k+t_{p}^{r s}(k)<k_{r s}^{*}-\Delta_{r s}, \\
\gamma\left[k+t_{p}^{r s}(k)-k_{r s}^{*}-\Delta_{r s}\right] \text { if } k+t_{p}^{r s}(k)>k_{r s}^{*}+\Delta_{r s}, \\
0 & \text { otherwise, }
\end{array}\right.
$$

where $\beta$ is the unit cost of schedule delay time-early, $\gamma$ is the unit cost of schedule delay time-late, and $\left[k_{r s}^{*}-\Delta, k_{r s}^{*}+\Delta\right]$ is the window of arrival times at destination $s$. Without the schedule delay penalty for travel from origin r. $k_{r s}^{*}$ is middle point of the time window and may represent the official work start time for trips between OD pair $r$. We assume $\gamma>\beta>\alpha$.

\subsection{Market penetration and compliance rate model}

We will derive a market penetration model and a compliance rate model. Firstly we assume that drivers' decision making structure about the decision of pur- chase of ATIS devices and the decision of compliance of ATIS advices regarding average travel cost saving can be represented as a tree structure with driver purchase choice of ATIS device at the upper level and drivers' compliance choice of ATIS advice at the lower level as shown Fig 1.

Drivers' purchase choice can be expressed as:

$$
p=1 \text { if } U_{p}>S_{p}, p=0 \text {, if } U_{p}<S_{p} .
$$

Drivers' compliance choice can be expressed as:

$$
a=1 \text { if } U_{a}>S_{a}, a=0 \text {, if } U_{a}<S_{a} .
$$

The above models considered here assume that a driver will adopt ATIS advice when the drivers' device purchase utility $U_{p}$ and compliance utility $U_{a}$ exceed certain thresholds $S_{p}$ and $S_{a}$. Where $p=1$ is the case that a driver will purchase a ATIS device, $p=0$ the case that a driver will not purchase a device, $a=1$ the case that a driver will adopt ATIS advice and $a=0$ the case a driver will not adopt ATIS advice.

Assuming that the error term has Gumbel distribution, drivers' device purchase utility $U_{p}$ and compliance utility $U_{a}$ threshold $S_{p}$ and $S_{a}$ can be given by the following equations:

$$
\begin{aligned}
& U_{p}=\varpi+\lambda_{1} V_{p}+\varepsilon_{p}^{u} ; \\
& S_{p}=\pi_{1}+\varepsilon_{p}^{s} ; \\
& U_{a}=\lambda_{2} C_{a v e}^{r s}+\varepsilon_{a}^{u} ; \\
& S_{a}=\pi_{2}^{r s}+\varepsilon_{a}^{s} ; \\
& V_{p}=\ln \left(\exp \left(C_{a v e}^{r s}\right)+\exp \left(\pi_{2}^{r s}\right)\right),
\end{aligned}
$$

where $\varpi$ is a general parameter capturing other benefits such as convenience of having the device, etc. $\pi_{1}$ is the deterministic terms of the thresholds which show the capital cost to buy and use an ATIS device annually. $\pi_{2}^{r s}$ shows the deterministic term of the threshold of the travel cost saving of the equipped and complied driver on OD pair rs. $\lambda_{1}, \lambda_{2}$ are the value of time, $\varepsilon_{p}^{u}, \varepsilon_{p}^{s}, \varepsilon_{a}^{u}, \varepsilon_{a}^{s}$ are the respective error terms; Travel cost saving, $C_{\text {ave }}^{r s}$, is defined as the difference of the average travel cost between complied vehicles and unequipped, non-complied vehicles on OD pair rs, expressed as:

$$
\begin{aligned}
& C_{\text {ave }}^{r s}=\sum_{p} \sum_{k} c_{p}^{r s}(k) \tilde{P}_{p}^{r s}(k)- \\
& \sum_{p} \sum_{k} c_{p}^{r s}(k) \hat{P}_{p}^{r s}(k) .
\end{aligned}
$$


The first term on the right side represents the average travel cost of the unequipped and non-complied drivers on each OD pair rs, the second term on the right side represents the average travel cost of the complied drivers on each OD pair rs.

The concurrent probabilities that a driver will purchase an ATIS device and adopt ATIS advice can be given by the nested logit model expressed as follows.

$$
\begin{aligned}
& P_{p a}^{r s}(p, a \mid p=1, a=1)= \\
& \frac{\exp \left(\varpi+\lambda_{1} V_{p}\right)}{\exp \left(\varpi+\lambda_{1} V_{p}\right)+\exp \left(\pi_{1}\right)} \times \\
& \frac{\exp \left(\lambda_{2} C_{\text {ave }}^{r s}\right)}{\exp \left(\lambda_{2} C_{\text {ave }}^{r s}\right)+\exp \left(\pi_{2}^{r s}\right)} .
\end{aligned}
$$

The number of drivers to purchase a ATIS device is determined by the following equation:

$$
P_{p}^{r s}=\frac{\exp \left(\varpi+\lambda_{1} V_{p}\right)}{\exp \left(\varpi+\lambda_{1} V_{p}\right)+\exp \left(\pi_{1}\right)}
$$

where $P^{r s}$ is the value of market penetration of ATIS for each ${ }^{p} \mathrm{OD}$ pair $r s$.

The compliance rate of ATIS is determined by the following equation.

$$
P_{a}^{r s}=\frac{\exp \left(\lambda_{2} C_{a v e}^{r s}\right)}{\exp \left(\lambda_{2} C_{a v e}^{r s}\right)+\exp \left(\pi_{2}^{r s}\right)}
$$

\section{A solution algorithm}

With the market penetration and compliance rate given by Eqs. (36) and (37), the resulting travel demand of complied drivers and non-complied and unequipped drivers are given as below:

$$
\begin{aligned}
& q_{1}^{r s}=q^{r s} \cdot\left(1-P_{p a}^{r s}(p, a \mid p=1, a=1)\right) ; \\
& q_{2}^{r s}=q^{r s} \cdot P_{p a}^{r s}(p, a \mid p=1, a=1) ; \\
& P_{p a}^{r s}(p, a \mid p=1, a=1)=P_{p}^{r s} \cdot P_{a}^{r s},
\end{aligned}
$$

where $q_{1}^{r s}$ expresses the demand of complied travelers between OD pair $r s . q_{2}^{r s}$ expresses the demand of non-complied and unequipped drivers between OD pair $r s . q^{r s}$ expresses total demand between OD pair $r s$. We firstly consider the solution of multi-user dynamic stochastic route and departure time equilibrium models for given $q_{1}^{r s}$ and $q_{2}^{r s} \quad r s \in R S$, then we present a procedure for the determination of the ATIS market penetration and compliance rate.
The algorithm assumes that a set of working paths is available in advance. Here we use a k-shortest path to enumerate a set of potential used paths. Alternatively, this set could be obtained from personal interviews and hence constitutes a set of likely used paths.

The multi-user dynamic stochastic route and departure time equilibrium algorithm can be described as follows:

\section{MU-DSRD-EA}

Step 0: initialization. Set initial iteration number $t=1$, choose initial path inflow rate $\hat{f}_{p}^{r s}(k)_{t}, \widetilde{f}_{p}^{r s}(k)_{t} \quad \forall r s, p, k$, and set $t=0$.

Step 1: Perform dynamic network loading to

compute the correspondent cost pattern.

$$
c_{p}^{r s}(k)_{t} \forall r s, p, k .
$$

Step 2: calculate an auxiliary path flow

$$
\hat{f}_{p}^{r s}(k)_{t}^{*}, \tilde{f}_{p}^{r s}(k)_{t}^{*} \forall r s, p, k \text { by Eq. (1) and (9). }
$$

Step 3: move. Find the new path flow pattern

$$
\begin{aligned}
& \hat{f}_{p}^{r s}(k)_{t+1}=\hat{f}_{p}^{r s}(k)_{t}+\gamma_{t}\left(\hat{f}_{p}^{r s}(k)_{t}^{*}-\hat{f}_{p}^{r s}(k)_{t}\right), \\
& \tilde{f}_{p}^{r s}(k)_{t+1}=\tilde{f}_{p}^{r s}(k)_{t}+\gamma_{t} \cdot\left(\tilde{f}_{p}^{r s}(k)_{t}^{*}-\tilde{f}_{p}^{r s}(k)_{t}\right) .
\end{aligned}
$$

Step 4: convergence criterion. If

$$
\sum_{p} \sum_{k}\left(\left|\hat{f}_{p}^{r s}(k)_{t+1}-\hat{f}_{p}^{r s}(k)_{t}\right|+\left|\tilde{f}_{p}^{r s}(k)_{t+1}-\tilde{f}_{p}^{r s}(k)_{t}\right|\right) \leq \varepsilon
$$

then stop, otherwise, go to step 1 and set $t=t+1$.

In the algorithm the step size $\gamma_{t}$ could be determined a priori. For example the following simplest move size sequences could be applied $\gamma_{t}=\frac{1}{t}$.

The algorithm for the determination of the ATIS market penetration and compliance rate is described as follows:

Step 0: determine an initial value of

$$
\begin{aligned}
& \quad P_{p a}^{r s(n)}(p, a \mid p=1, a=1) \quad\left(\text { Written as } P_{p a}^{r s(n)}\right. \text { as } \\
& \text { below) and set } n=0 .
\end{aligned}
$$

Step 1: perform MU-DSRD-EA to get

$$
C_{\text {ave }}^{r s}, \forall r s \in R S \text {. }
$$

Step 2: update $P_{p a}^{r s(n)}$ by Eq. (35), obtain new $P_{p a}^{r s(n+1)}$. Meanwhile, calculate the market penetration and compliance rate by Eqs. (36)-(37).

Step 3: convergence criterion.

If $\sum_{r s}\left|P_{p a}^{r s(n+1)}-P_{p a}^{r s(n)}\right| \leq \varepsilon$, then stop. Otherwise go to

\section{A numerical example}

We choose a simple network for a numerical example. The network scenario is shown in Fig 2, 12 links and 1 OD pair. The parameters of link travel 
time functions are also given in this figure. The link travel time function is assumed as follows: $t_{a}(k)=\chi_{a}+\alpha_{a} \cdot x_{a}(k)+\beta_{a} \cdot u_{a}(k)$, where $\chi_{a}$ is the free flow travel time on link $a$. $\alpha_{a}$ and $\beta_{a}$ are the parameters of the link travel time function. We consider a morning peak. We set $T$ be from 6 to 10 a.m. and $K=100$, so $\delta=2,4 \mathrm{~min}$. The ATIS and route choice parameters are: $\lambda_{1}=0.5, \lambda_{2}=0,5, \Phi=0,01$, $\pi_{1}=3,5, \pi_{2}=1,75$. Other data are: $a=6,4, \beta=3,9$, $\gamma=15,21, \Delta=0,2 \mathrm{~h}, k^{*}=9,0 \mathrm{~h}$.

We firstly examine the convergence of the iterative algorithm. Fig 2 plots the solution of the market penetration against iteration number, where the value of travel cost variability parameter $\tilde{\theta}$ for non-complied and unequipped drivers is fixed as 0,01 and values of $\hat{\theta}$ for complied drives and total travel demand $q^{\text {rs }}$ vary as shown in the legend. It can be observed that the iteration of the algorithm has a fast convergence; convergence is achieved in about four iterations in all cases. It seems that the level of demand and the degree of travel cost uncertainty have little impacts on the convergence of the algorithm.

Fig 3 gives the solution of the compliance rate. Similar convergence properties have been observed in other cases. Note that the convergence of the algorithm cannot be always guaranteed theoretically. Similar to Yang (1998), Ying (2003) non-convergence has been also observed in our examples where the algorithm may oscillate between two points when demand and values of $\hat{\theta}$ are higher.

Fig 4 depicts the change of the equilibrium market penetration and compliance rate against the value $\hat{\theta}$ of parameter with parameter $\tilde{\theta}$ fixed to be 0.01 . Total travel demand $q^{\mathrm{rs}}$ varies as shown in the legend. As aforementioned, the value of $\hat{\theta}$ shows the degree of equipped and complied traveler's uncertainty on travel cost, and also implies the quality of the provided information. As parameter $\hat{\theta}$ increases, complied drivers have better perception of travel cost due to higher information quality provided by ATIS (Fig 5), and thus the market penetration and compliance rate will increase. This numerical example confirms the previous studies of the traveler's purchase behavior and compliance behavior made by Ying (2003), etc. The difference with Ying (2003) is mainly that the magnitude of travel demand has similar impacts on the level of market penetration and compliance rate because both mainly depend on the quality of ATIS information.

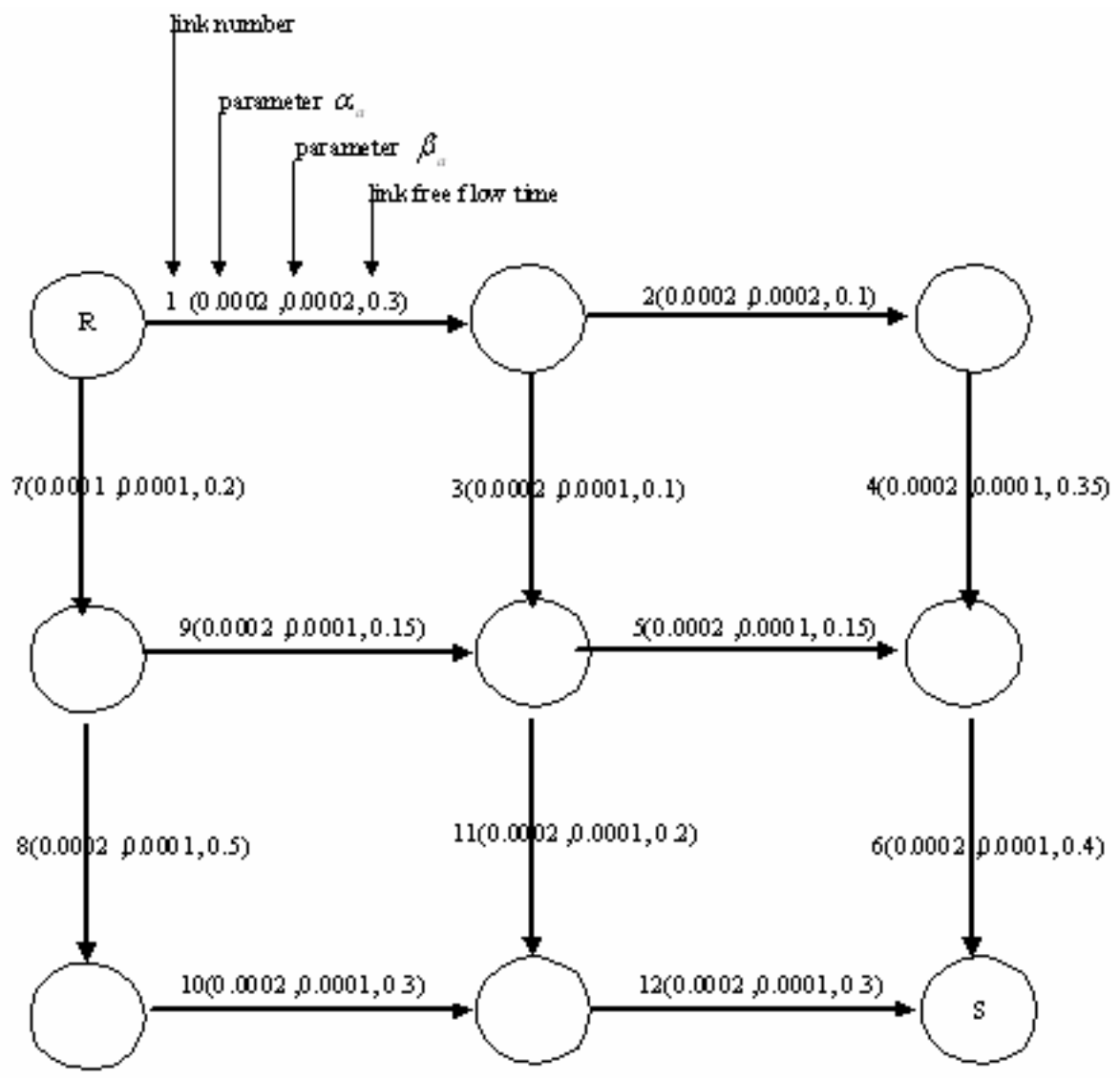

Fig 2. Example network 


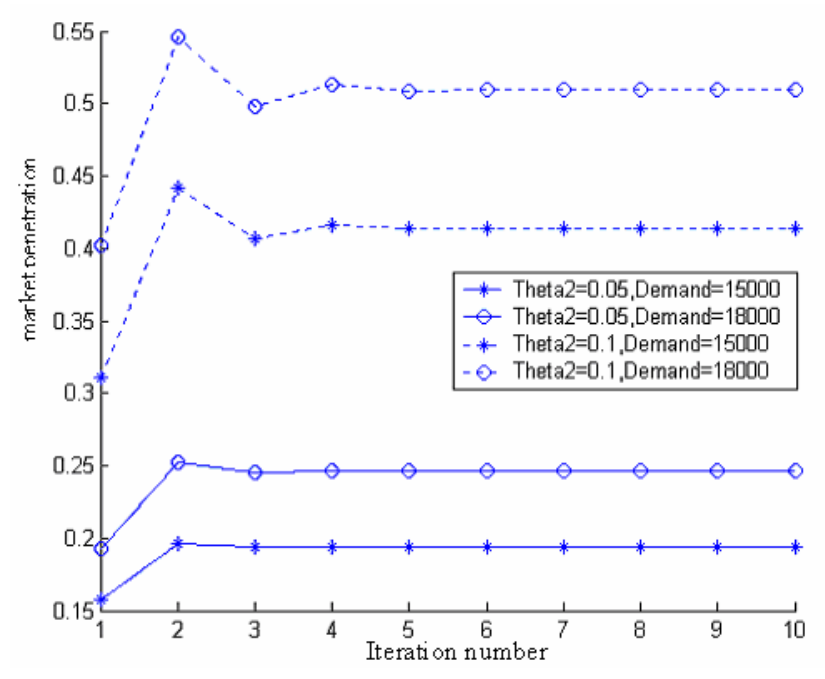

Fig 3. Convergence of the outer iteration of the algorithm for different Demand and Theta2

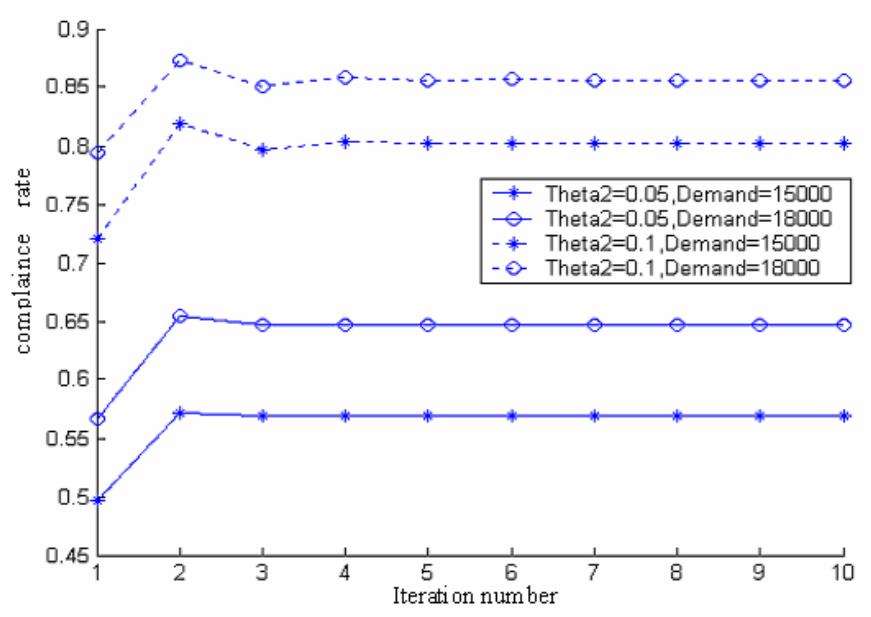

Fig 4. Convergence process of compliance rate for different Demand and Theta2

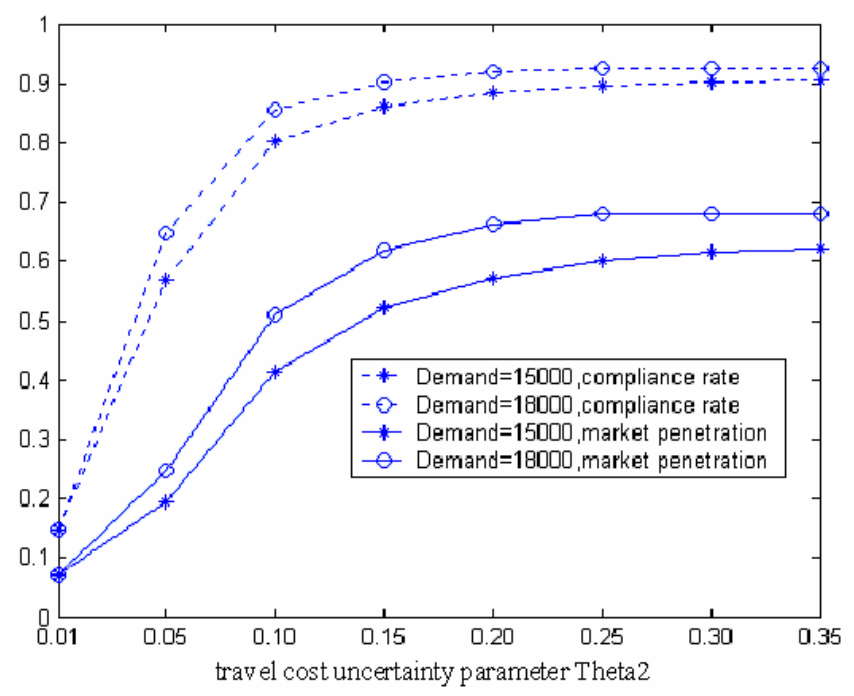

Fig 5. Effects of travel cost uncertainty of complied travelers on market penetration and compliance rate of ATIS

\section{Conclusion}

In this paper we propose a multi-user dynamic stochastic route and departure time equilibrium model with the equilibrium market penetration and compliance rate in ATIS environment. The nested-logit model is given for modelling the driver purchase behavior and compliance behavior. The proposed iterative algorithm is applied to a numerical example and demonstrated to have a fast convergence in general similar to a static example.

In future studies the proposed model could be extended in several directions. Firstly, this model assumes that the total demand over the network is fixed. To consider variable demand is one direction. Secondly, the proposed model does not consider or produce system optimal flow patterns how to combine ATIS with a congestion toll model so as to achieve a system optimal pattern would be another important research direction.

\section{References}

1. Abdel Aty, M. A., Kitamura, R. and Jovanis, 1997. Using stated preference data for studying the effect of advanced traffic information on drivers' route choice. Transportation Research C(5)-1, 39-50.

2. Ben-Akiva, M.; De Palama, A.; Kaysi, I., 1991. Dynamic network models and driver information systems. Transportation Research 25A, 251-266.

3. Benneet, L., 1993. The existence of equivalent mathematical programs for certain mixed equilibrium traffic assignment problems. European Journal of Operational Research 71, p. 177-187.

4. E. Hato, etc, 1999. Incorporating an information acquisition process into a route choice model with multiple information sources, Transportation Research C, Vol 7, p. 109-129.

5. Emmerink, R.; Axhausen, K.; Nijkamp, P., Rietveld, P., 1995. Effects of information in road transport networks with recurrent congestion. Transportation 22, p. 21-53.

6. Kanafani, A.; Al-Deek, H., 1991. A simple model for route guidance benefit. Transportation Research 25B, p. 191-202.

7. Tsuji, H.; Takahashi, R.; Kawashima, H., 1985. A stochastic approach for estimating the effectiveness of route guidance strategies. Transportation Science 19, p. 333-351.

8. Mahmassani, H. S. 1994. Development and testing of dynamic traffic assignment and simulation procedures for ATIS/ATMS application. Technical Report DTFH6 1-90-R-00074-FG. Center for transportation Research, The University of Texas at Austin.

9. Mahmassani, H. S.; Liu, Y. H., 1999. Dynamics of commuting decision behaviour under advanced traveler information systems. Transportation Research C7, p. 91107.

10. Hong K. Lo; Ran, B.; Hongola, B., 1996. Multi-class dynamic traffic assignment model: Formulation and 
computational experiences. Transportation Research Records 1537, p. 74-82.

11. Lo, H. and Szeto, W. Y. 2002a, a methodology for sustained traveler information's services, Transportation Research B, Vol 36, p.113-130.

12. Lo, H. and Szeto, W. Y. 2002b, Paradigms of modelling advanced traveler information services. In: Proceedings of IEEE $5^{\text {th }}$ conference on ITS.

13. Yang, H etc, 1993. Exploration of route choice behavior with advanced travel information using neural network concept. Transportation 20, p. 199-223.

14. Yang, H., 1998. Multiple equilibrium behavior and advanced traveler information systems with endogenous market penetration. Transportation Research 32B, p. 205218.

15. Yang, H., 1999. Evaluating the benefit of a combined route guidance and road pricing system in a network with recurrent congestion. Transportation 26, p. 299-322.

16. Yang, H.; Meng, Q., 2001. Modelling user compliance of advanced traveler information systems: dynamic evolution and stationary equilibrium. Transportation Research 35A, p. 895-912.
17. Yafeng Yin, Hai Yang, 2003 Simultaneous determination of the equilibrium market penetration and compliance rate of advanced traveler information systems. Transportation Research, Part A 37, p. 165-181.

18. Ran, B.; Lo, H. K.; Boyce, D. E., 1996. A formulation and solution algorithm for a multi-class dynamic traffic assignment model. In: Lesort, J.-B. (Ed.), Transportation and Traffic Theory. Pergamon, Oxford, p. 195-216.

19. Reddy, P.; Yang, H, etc. 1995. Design of an artificial simulator for analyzing route choice behavior in the presence of information system. Mathematical and Computer modelling 22, p. 119-147.

20. Van Vuren T \& Watling DP, 1991. A multiple user class assignment model for route guidance. Transportation research record 1306, p. 22-31.

21. Williams and Huang, 2003. A multi-class dynamic user equilibrium model for queuing networks with advanced traveler information systems. Submitted for Transportation Research C. 